\title{
Carcinoma hepatocelular variedad fibrolamelar metastásico en menores de 20 años. Reporte de 2 casos tratados con intención curativa y revisión de la literatura
}

\author{
Jean M Butte ${ }^{1}$, Enrique W augh ${ }^{1,2}$, Manuel Meneses ${ }^{1,2}$, \\ Rossana Pruzzo ${ }^{1}$, Cristián Carvallo ${ }^{2}$, Francisca Redondo ${ }^{1}$, \\ Claudio Suárez ${ }^{2}$, Hugo Parada ${ }^{1,2}$, Horacio Amaral ${ }^{1}$, \\ Hernán de La Fuente ${ }^{1,2}$.
}

\section{Fibrolamellar liver carcinoma. Report of two cases}

\begin{abstract}
Fibrolamellar bepatocellular carcinoma (FLC) is a rare bistologic variant of hepatocellular carcinoma that appears most commonly in teenagers and young adults. The diagnosis is often made incidentally and surgical resection is the only curative treatment. Here we report two cases of incidental FLC involving a 19 year-old male, initially diagnosed with screening abdominal ultrasound, and a 14 year-old female that presented with abdominal pain. Diagnostic workup consisted of abdominal PET/CT and MRI Imaging studies and tissue diagnosis was confirmed with percutaneous liver biopsy. Both patients were treated with radical liver resection/tumor excision. However, tumor recurrence was observed in both during short-term follow-up. The male patient was treated successfully with surgical treatment however the female patient succumbed to pregression of disease (Rev Méd Chile 2009; 137: 394-400).
\end{abstract}

(Key words: Carcinoma, hepatocellular; Hepatectomy; Liver neoplasms)

Recibido el 13 de marzo, 2008. Aceptado el 26 de septiembre, 2008.

${ }^{1}$ Instituto Oncológico Fundación Arturo López Pérez y ${ }^{2}$ Clínica Santa María, Santiago, Chile.

$\mathrm{L}$

a variedad fibrolamelar del carcinoma hepatocelular es poco frecuente en la población general, ya que representa menos de $1 \%$ de todos los casos de carcinoma hepatocelular ${ }^{1}$. Se presenta fundamentalmente en adolescentes y adultos jóvenes como una masa tumoral hepática única, frecuentemente de gran tamaño, no asociada a

$\overline{\text { Correspondencia } a \text { : Dr. Hernán de La Fuente. Fundación }}$ Arturo López Pérez. Rancagua 878, Santiago, Chile. Teléfono: 56-2-4457247. Fax: 56-2-4218597.

E mail: delafueh@yahoo.com cirrosis y rodeada de una cápsula fibrosa. Su diseminación es preferentemente a través de metástasis linfáticas regionales ${ }^{2,3}$. En nuestro país sólo se han comunicado casos aislados ${ }^{4}$.

El diagnóstico en la mayoría de los pacientes se realiza en forma incidental y en etapas avanzadas de la enfermedad 5 . Pese a esto, el pronóstico es más favorable que el del carcinoma hepatocelular habitual. En los casos que ya presentan enfermedad metastásica, el tratamiento queda supeditado a la factibilidad técnica de lograr resecciones quirúrgicas completas ${ }^{6}$. 
Nuestro objetivo es presentar dos casos clínicos de pacientes menores de 20 años tratados en forma quirúrgica por un carcinoma hepatocelular variedad fibrolamelar, metastásicos al momento del diagnóstico.

\section{Casos Clínicos}

Caso 1. Hombre de 19 años sin antecedentes mórbidos. En diciembre de 2005 se detectó una lesión tumoral sólida en el lóbulo hepático derecho, en una ecografía abdominal realizada como parte de un examen de salud para deportistas de élite. Los niveles de alfafetoproteína y CA 19-9 fueron normales y la serología para virus de hepatitis B y C negativa. Una tomografía computada de abdomen y pelvis ratificó la presencia de una lesión hepática sólida de 7 × $5 \mathrm{~cm}$, con refuerzo

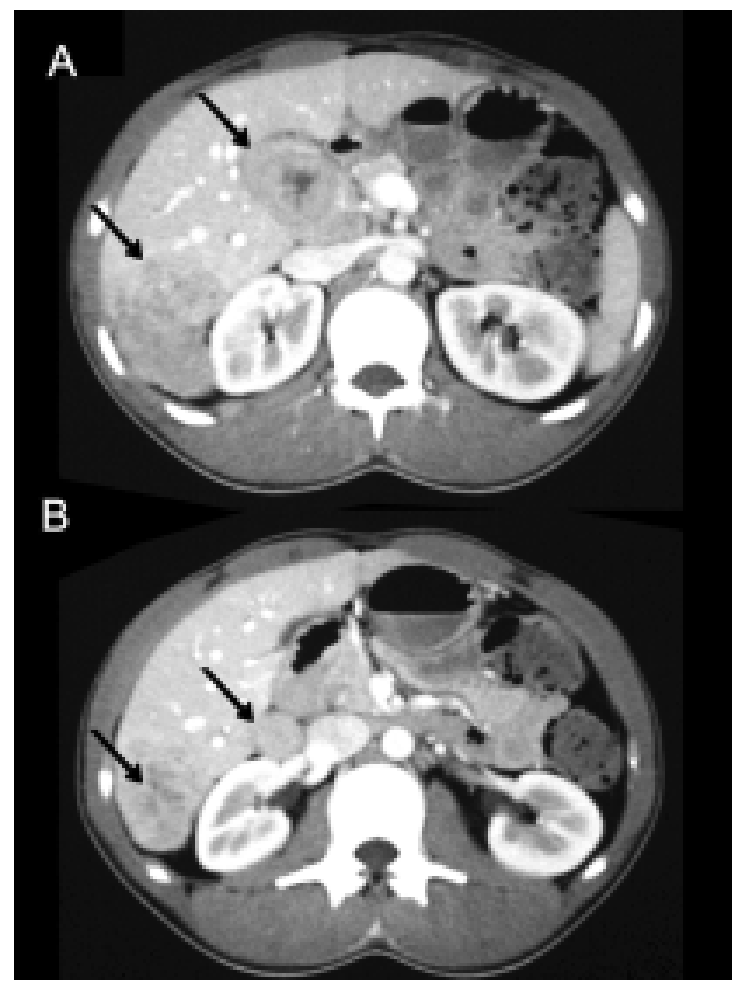

Figura 1. Tomografía computada de abdomen y pelvis. A) Tumor del lóbulo hepático derecho (flecha), asociado a adenopatías en el hilio hepático (flecha). B) Visión más inferior del tumor del lóbulo hepático derecho (flecha), asociado a una adenopatía perirrenal (flecha). periférico y calcificación central en los segmentos posteriores del lóbulo derecho, asociada a una lesión satélite de $12 \mathrm{~mm}$, de características similares y linfonodos de aspecto patológico en el hilio hepático, sugerente de un carcinoma hepatocelular variedad fibrolamelar (Figuras 1a y 1b). La resonancia magnética de abdomen y pelvis confirmó un tumor sólido de $7 \mathrm{~cm}$, hipervascular y con pseudocápsula. La lesión era hipointensa en T1 y presentaba focos centrales de hiposeñal en T2. Además se observó un nódulo satélite de $2 \mathrm{~cm}$ y linfonodos de hasta $5 \mathrm{~cm}$ en el ligamento hepatoduodenal y en el retroperitoneo supra e infrarrenal hasta la bifurcación aórtica. El diagnóstico radiológico inicial fue compatible con un colangiocarcinoma hepático o un carcinoma hepatocelular variedad fibrolamelar (Figura 2). La tomografía computada de tórax mostró 2 nódulos menores de $1 \mathrm{~cm}$ en el pulmón izquierdo que se interpretaron como inespecíficos. Con el fin de precisar el diagnóstico de la lesión hepática se realizó una biopsia percutánea, cuyas características anatomopatológicas fueron compatibles con un carcinoma hepatocelular variedad fibrolamelar. Se complementó el estudio con una tomografía de positrones con ${ }^{18} \mathrm{~F}$-deoxiglucosa (PET) que mostró leve a moderado hipermetabolismo en el lóbulo hepático derecho (segmentos VI y VII) con un SUV máximo de $3,3 \mathrm{~g} / \mathrm{ml}$, leve hipermetabolismo en las adenopatías del hilio hepático con un SUV máximo de $2,8 \mathrm{~g} / \mathrm{ml}$ y leve hipermetabolismo en una adenopatía paratraqueal con un SUV máximo de 1,6 g/ml (Figura 3).

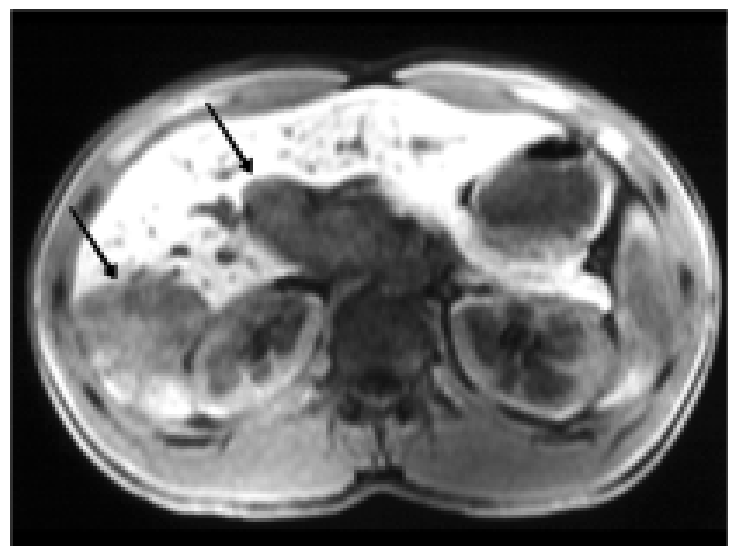

Figura 2. Resonancia magnética de abdomen y pelvis (T1). Tumor del lóbulo hepático derecho asociado a adenopatía del hilio hepático (flechas). 


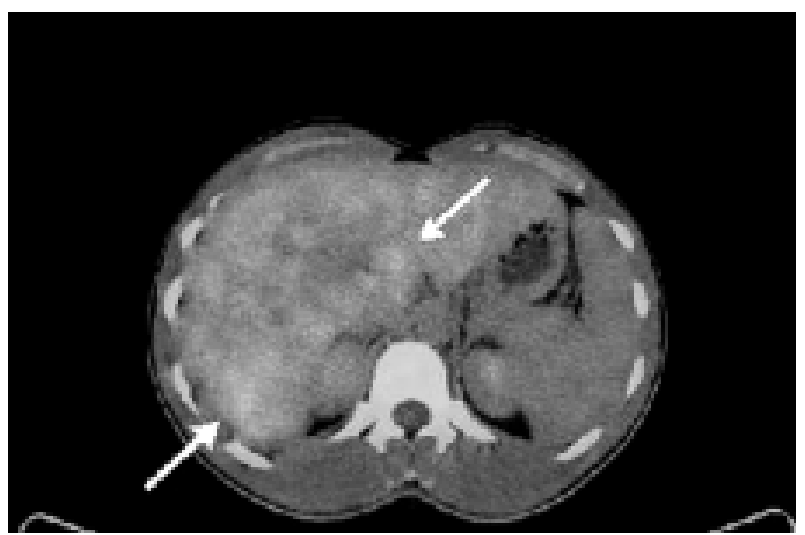

Figura 3. PET/CT preoperatorio. Tumor del lóbulo hepático derecho asociado a adenopatía del hilio hepático (flechas), con hipermetabolismo moderado.

Durante diciembre y enero de 2006 fue evaluado por distintos equipos quirúrgicos de diferentes centros nacionales donde se descartó el tratamiento quirúrgico por presentar enfermedad metastásica intrahepática y retroperitoneal. Por esta razón se decidió administrar quimioterapia paliativa y recibió 6 ciclos de cisplatino, doxorrubicina y bevacizumab.

En junio de 2006, después de finalizar el tratamiento quimioterápico fue reevaluado con un nuevo PET-CT. En éste se observó que la lesión hepática presentaba las mismas características topográficas, pero había disminuido discretamente su metabolismo (descenso de SUV máximo de 3,3 a $2,5 \mathrm{~g} / \mathrm{ml}$ ) y las lesiones del hilio hepático y retroperitoneo no habían presentado variación en sus características morfológicas ni en su captación. Se hizo más evidente en este examen la presencia de un implante peritoneal sobre la cápsula del riñón derecho que en la visión retrospectiva ya se hallaba presente en los estudios de imágenes previos.

Debido a la estabilización de las lesiones, y luego de la evaluación por el equipo de Cirugía Oncológica Digestiva, se decidió la exploración quirúrgica para determinar la factibilidad de una resección oncológica completa. En la cirugía se confirmó la presencia de una masa hepática de 8 x $6 \mathrm{~cm}$ en el lóbulo hepático derecho con lesiones satélites periféricas e indemnidad de parte del lóbulo izquierdo (segmentos 2 y 3). Además eran evidentes nódulos tumorales en el peritoneo diafragmático derecho y sobre la cápsula renal derecha, así como linfonodos de aspecto tumoral en el hilio hepático, ligamento hepatoduodenal y retroperitoneo supra e infrarrenal. No había carcinomatosis inframesocólica y todas las lesiones parecieron abordables para una resección extendida. Fue sometido a una hepatectomía derecha extendida (segmentos 4-5-6-7- y 8), peritonectomía diafragmática y de la cápsula renal derecha y disección linfática extensa que incluyó el ligamento hepatoduodenal y el retroperitoneo en sus cuatro cuadrantes supra e infrarrenales. El paciente evolucionó sin complicaciones y fue dado de alta el día 11 postoperatorio.

El estudio anatomopatológico mostró un carcinoma hepatocelular variedad fibrolamelar de $8 \mathrm{~cm}$, asociado a lesiones satélites peritumorales y permeaciones tumorales vasculares linfáticas. El parénquima hepático no tumoral presentaba inflamación portal reactiva, linfoide e inespecífica. Los bordes quirúrgicos se encontraron libres de tumor. Se observó metástasis en 3 nódulos del peritoneo diafragmático, en un linfonodo adyacente a la vena cava (1/1), en 2 linfonodos periportales $(2 / 2)$ y en una masa de linfonodos retroperitoneales (2/8).

En el control de imágenes realizado en agosto de 2007 con un PET-CT, se detectaron 2 lesiones compatibles con sitios puntuales de recurrencia en el retroperitoneo. Se observó una lesión de $5 \mathrm{~cm}$ por detrás de la vena renal izquierda y otra lesión de $2 \mathrm{~cm}$ en la cápsula renal posterior derecha adherida a la pared costal. El metabolismo de estas lesiones se encontraba aumentado (SUV máximo de 2,9 y $2,5 \mathrm{~g} / \mathrm{ml}$, respectivamente) (Figura 4). En octubre de 2007 fue explorado quirúrgicamente, se identificaron y resecaron ambas lesiones y se 


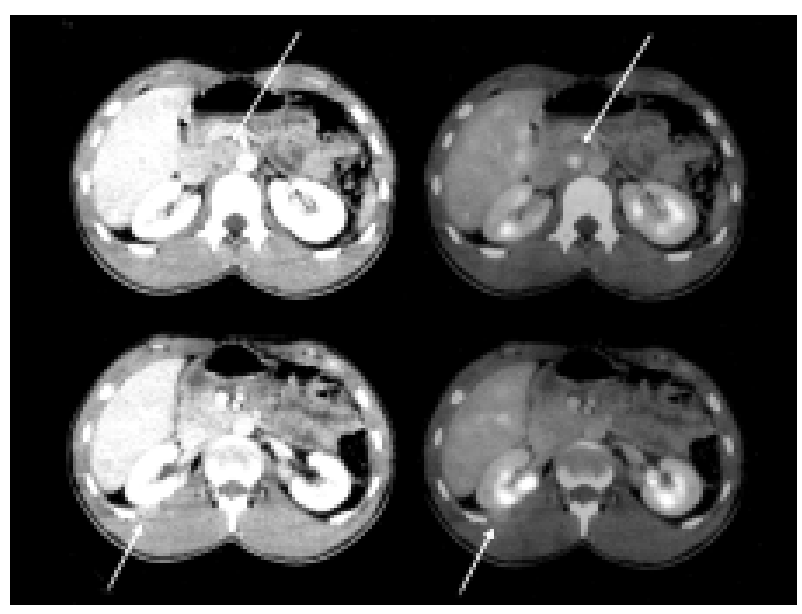

Figura 4. PET/CT realizado como control postoperatorio. Se observan dos sitios de recurrencias retroperitoneales (flechas). Una lesión de $5 \mathrm{~cm}$ por detrás de la vena renal izquierda y otra lesión de $2 \mathrm{~cm}$ en la cápsula renal posterior derecha adherida a la pared costal. Ambas con aumento del metabolismo.

demostró la ausencia de otros sitios de recurrencia abdominal. El estudio anatomopatológico contemporáneo mostró que las dos lesiones fueron resecadas en forma completa y los bordes quirúrgicos se encontraban libres de tumor. El estudio anatomopatológico definitivo confirmó la resección completa de ambas lesiones, diagnosticadas como metástasis de carcinoma hepatocelular variedad fibrolamelar moderadamente diferenciado con necrosis y presencia de compromiso linfovascular. El paciente fue dado de alta al día 5 postoperatorio y se reintegró a su vida habitual sin problemas a las tres semanas de la exploración. Actualmente se mantiene asintomático, en controles regulares.

Caso 2. Mujer de 14 años sin antecedentes mórbidos. Consultó por un cuadro clínico de 1 año de evolución, caracterizado por dolor abdominal epigástrico inespecífico y baja de peso. Se realizó una ecografía abdominal que mostró una lesión tumoral sólida en el lóbulo hepático derecho. La tomografía computada de abdomen y pelvis confirmó la presencia de una lesión sólida de $8 \mathrm{~cm}$ en el lóbulo hepático derecho asociada a lesiones satélites bilaterales sin signos de daño hepático crónico. Además se observaron múltiples linfonodos de aspecto patológico en el ligamento hepatoduodenal, arteria hepática, tronco celíaco y retroperitoneales. La tomografía computada de tórax fue normal. El estudio de virus hepatitis $\mathrm{B}$ y $\mathrm{C}$ fue negativo y los niveles de alfafetoproteína y CA 19-9 fueron normales. Se realizó una biopsia percutánea de la masa hepática principal que fue compatible con un carcinoma hepatocelular variedad fibrolamelar.
Se complementó el estudio con un PET que mostró un marcado aumento de volumen del lóbulo hepático derecho, que se encontraba extensamente infiltrado por una masa de $12 \times 16 \mathrm{~cm}$ y que presentaba un intenso hipermetabolismo heterogéneo de ${ }^{18} \mathrm{~F}$-deoxiglucosa (SUV máximo de 6,8 ). Además se observaron linfonodos de gran tamaño en el hilio hepático, ligamento hepatoduodenal y región intercavoaórtica que midieron 55, 23, 50, 42, $38 \mathrm{~mm}$, y que también mostraron hipercaptación de la glucosa marcada. Al mismo tiempo se detectó un nódulo pulmonar hipermetabólico de $11 \mathrm{~mm}$ en el lóbulo superior izquierdo que se encontraba en contacto con la vena pulmonar superior izquierda y que no había sido detectado en los estudios previos. En el lóbulo medio del pulmón derecho se observó otro nódulo de $3 \mathrm{~mm}$ que no presentó captación del trazador. Se decidió la exploración quirúrgica abdominal de manera inicial. En caso de lograr resección completa el plan terapéutico continuaría con exploración pulmonar y resección de las metástasis pulmonares en un segundo tiempo quirúrgico.

Durante la operación (30/06/2007) se observó que el lóbulo hepático derecho se encontraba completamente reemplazado por tumor y en el lóbulo izquierdo existían 6 metástasis hepáticas repartidas en los segmentos 2-3 y 4 , resecables con la resección mayor y metastasectomías. En el ligamento hepatoduodenal y en el retroperitoneo había metástasis nodales múltiples con desplazamiento de la vena cava, el riñón derecho y los vasos renales hacia anterior. No había carcinomatosis peritoneal. Fue sometida a una hepatectomía derecha extendida (segmentos 4-5-6-7 y 8), resec- 
ción de las metástasis de los segmentos 2 y 3, así como, disección linfática del ligamento hepatoduodenal y del retroperitoneo. Se logró una resección macroscópica completa de todas las lesiones abdominales (Figura 5A). Al cuarto día postoperatorio presentó una fístula biliar externa de bajo débito que se trató en forma conservadora y fue dada de alta al día 14 postoperatorio.

El estudio anatomopatológico mostró un carcinoma hepatocelular variedad fibrolamelar moderadamente diferenciado de $14,5 \mathrm{~cm}$ (Figura $5 \mathrm{~B}$, C, D), asociado a permeaciones tumorales vasculares y linfáticas. El parénquima hepático no tumoral presentaba cambios portales reactivos y no había cirrosis. En el lóbulo hepático izquierdo (segmentos 2 y 3) se observaron 6 metástasis (entre 0,2 y 1,4 cms). Todos los bordes estuvieron libres de tumor. Se observaron metástasis ganglionares en el ligamento hepatoduodenal, periportales y en el retroperitoneo que formaban conglomerados tumorales de $5,5,7,0$ y $9,0 \mathrm{~cm}$.

Una tomografía computada de tórax realizada en agosto de 2007 mostró progresión evidente de las lesiones pulmonares en el pulmón izquierdo. Fue intervenida quirúrgicamente comprobándose la presencia de múltiples metástasis pulmonares y pleurales que fueron resecadas. El estudio anatomopatológico confirmó 7 metástasis pulmonares (entre 0,4 y 0,9 cms) y 11 metástasis pleurales (la mayor de $0,7 \mathrm{cms}$ ) asociadas a permeaciones vasculares tumorales. Todos los bordes quirúrgicos estuvieron libres de tumor. Evolucionó sin complicaciones y fue dada de alta al $4^{\circ}$ día postoperatorio.

Dos meses después de la última operación la paciente se encontraba asintomática. Sin embargo, en el control de imágenes aparecieron nuevas lesiones en el hígado remanente, pulmonares bilaterales y mediastínicas, no susceptibles de tratamiento resectivo.

La paciente falleció en enero de 2008, debido a progresión de la enfermedad.

\section{DisCUSIÓN}

La variedad fibrolamelar del carcinoma hepatocelular es poco frecuente en la población general. En un estudio poblacional que abarcó un periodo de 15 años, se encontraron 68 casos de esta

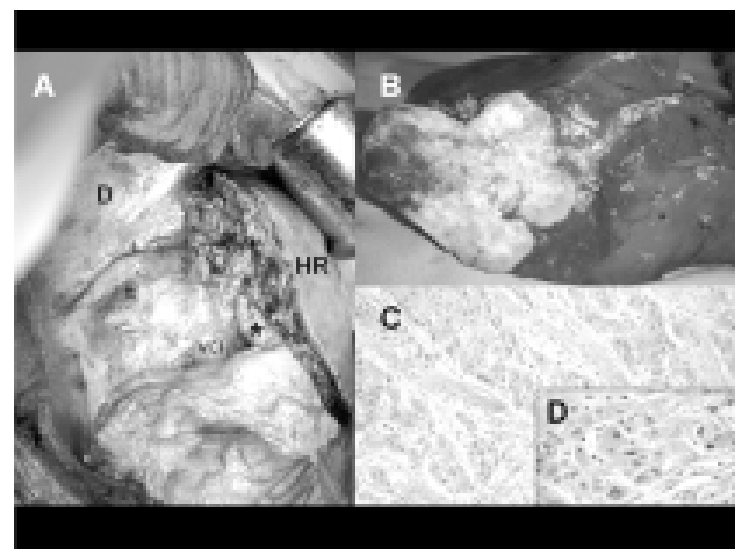

Figura 5. A) Hepatectomía derecha extendida, disección linfática del ligamento hepatoduodenal y retroperitoneal. $\mathrm{D}$ =diafragma, $\mathrm{HR}$ =hígado remanente, $\mathrm{VCI}$ =vena cava inferior, ${ }^{*}=$ pedículo hepático. B) Sección de la pieza quirúrgica de hepatectomía extendida, que muestra corte de lesión tumoral de bordes lobulados y límites bien definidos, con áreas de hemorragia más otras de aspecto fibroso. La lesión neoplásica alcanzo 14,5 cm en diámetro mayor. C) Tinción de hematoxilina eosina (10x) muestra una neoplasia de células poligonales de gran tamaño obtenidas del caso N.2 donde las trabéculas se encuentran separadas por bandas de colágeno, focalmente grueso. D) Tinción de hematoxilina eosina (40x) muestra un detalle de la citología de la neoplasia donde se observa el tamaño celular y nuclear, con hipercromasia y presencia de nucléolos de gran tamaño.

variedad tumoral entre 7.896 enfermos con un carcinoma hepatocelular típico, lo que representó $0,9 \%$ de los casos ${ }^{7}$.

A diferencia de lo observado en pacientes con un carcinoma hepatocelular típico, esta variedad tumoral ha sido descrita fundamentalmente en adolescentes y adultos jóvenes (edad promedio cercana a los 30 años) y no existen diferencias según el género, a diferencia del carcinoma hepatocelular que es 4 a 8 veces más frecuente en los hombres ${ }^{3}$. Katzenstein y $\operatorname{cols}^{8}$ revisaron la literatura mundial hasta el año 2003 y sólo encontraron 82 casos publicados en menores de 21 años.

$\mathrm{Al}$ igual que lo observado en nuestros pacientes, la mayoría de los enfermos consultan por dolor abdominal inespecífico y el diagnóstico se realiza en forma incidental. En una serie de 31 
enfermos evaluados con resonancia magnética se observó que $77 \%$ tuvo márgenes tumorales bien definidos, $68 \%$ presentó calcificaciones, $65 \%$ linfadenopatias y $71 \%$ una cicatriz central. Esta última característica es importante en la diferenciación con una hiperplasia nodular focal o con un hemangioma. Los enfermos con un tumor fibrolamelar presentan leve atenuación de la cicatriz central en $\mathrm{T} 2$, mientras que los con una hiperplasia nodular focal tienen una gran atenuación ${ }^{9,10}$.

En los últimos años la tomografía de positrones con ${ }^{18}$ F-deoxiglucosa ha sido útil como método de etapificación o de seguimiento de los tumores hepáticos, tal como se evidenció en el manejo de nuestros casos. Sin embargo, en nuestro país, el número de enfermos estudiado con este examen es bajo, posiblemente porque es un estudio que no está al alcance de todos los pacientes por disponibilidad y $\operatorname{costo}^{11}$.

Al igual que lo observado en estos dos enfermos, y a diferencia del carcinoma hepatocelular típico, los pacientes con una variedad fibrolamelar habitualmente cursan con niveles normales de alfafetoproteína y no presentan infección viral crónica ni daño hepatocelular crónico $^{12}$.

El rol de la biopsia percutánea por aguja fina es controversial. En estos pacientes fue útil y diagnóstica. Sin embargo no siempre se logra obtener un diagnóstico certero preoperatorio. Stipa y cols $^{3}$ realizaron una biopsia percutánea previo a la operación a 9 pacientes de 28 resecados. Sólo en 5 de ellos se confirmó un carcinoma hepatocelular variedad fibrolamelar. Desde el punto de vista patológico el carcinoma hepatocelular variedad fibrolamelar es un tumor con células poligonales con citoplasma de aspecto granular, denominado "oncocítico". Están separadas en grupos, filas o en forma individual por fibrosis en bandas fibrosas hialinas gruesas así como también más delicadas y con aumento de fibras de reticulina. La naturaleza granular del citoplasma de las células tumorales está dado por el gran número de mitocondrias que contienen, que son de tamaño y forma normales. Los núcleos tumorales son habitualmente de gran tamaño con nucléolo eosinofílico. Las figuras mitóticas no son abundantes ${ }^{13}$.

Hay consenso en que la resección quirúrgica completa es el único tratamiento con intención curativa que puede lograr sobrevidas alejadas 3,14 . La sobrevida a su vez, tiene directa relación con la obtención de márgenes libres de tumor y la resección completa de todas las lesiones ${ }^{5}$. La resecabilidad en cada paciente está determinada fundamentalmente por la extensión tumoral y la capacidad del equipo quirúrgico ${ }^{3}$. Sin embargo, se ha observado que la tasa de resecabilidad del carcinoma hepatocelular variedad fibrolamelar es mayor que la del carcinoma hepatocelular habitual (68\% vs 37\%) aunque se encuentre en etapa metastásica. Más de $50 \%$ de los pacientes requieren resecciones hepáticas mayores como hepatectomías derecha o izquierda y cerca de $20 \%$ una hepatectomía extendida asociada o no a resecciones segmentarias. Al momento de la cirugía, la mitad de los pacientes presentan compromiso tumoral nodal, 36\% compromiso vascular (la mayoría microscópico) y cerca de $20 \%$ puede presentar márgenes de resección comprometidos ${ }^{3}$.

El pronóstico y la sobrevida a 5 años es muy superior a la de los enfermos con un carcinoma hepatocelular típico (31,8\% vs 6,8\%). La sobrevida libre de recurrencia también es mayor y cercana a los 33 meses, sin embargo, se ha observado recurrencia incluso después de 10 años de tratamiento.

Los principales factores pronósticos negativos después de una resección serían la presencia de múltiples tumores, la invasión vascular (micro o macroscópica), el compromiso linfonodal y la resección R1 o R2. Los pacientes con linfonodos negativos tienen una sobrevida cercana a $100 \%$ a 5 años y de $20 \%$ a 10 años y los con linfonodos positivos presentan una sobrevida de $45 \%$ a 5 años $3,6-8$.

Al igual que lo observado en estos dos enfermos, más de la mitad de los pacientes presentarán recurrencia local o sistémica durante el seguimiento. En la mayoría ocurre en el hígado remanente, linfonodos, peritoneo o pulmón. El mejor tratamiento es la re-resección, lo que es factible en $20 \%$ a $60 \%$ de ellos. En el estudio de Stipa y cols ${ }^{3}, 17$ enfermos (61\%) necesitaron una segunda operación por recidiva tumoral en un intervalo promedio de 37 meses después de la primera resección, la sobrevida promedio después de la segunda operación fue de 26 meses. En pacientes con compromiso recurrente del hígado residual como foco único de enfermedad persistente no se ha demostrado que el trasplante hepático proporcione una mayor sobrevida. 
Tampoco se ha demostrado la utilidad de la quimioterapia como tratamiento neoadyuvante, adyuvante o paliativo y su uso no es estándar. Un estudio multicéntrico ${ }^{8}$ evaluó a 56 pacientes menores de 21 años, de los cuales 10 tenían un carcinoma hepatocelular variedad fibrolamelar. Fueron randomizados a recibir 4 ciclos de cisplatino, vincristina y 5 fluorouracilo (5-FU) o a recibir cisplatino y doxorrubicina. Al completar los 4 ciclos fueron reevaluados y se decidió en los enfermos sin respuesta, continuar con 4 ciclos más o ser tratados en forma quirúrgica. Después de los 8 ciclos sin respuesta se declaró al enfermo como no resecable y se decidió dar sólo tratamiento paliativo. Al analizar los esquemas empleados, no se observó diferencia en la respuesta entre los grupos de tratamiento. Tampoco mejoró la tasa de resecabilidad ni la sobrevida final. Patt y cols $^{15}$ comunicaron 9 enfermos con un carcinoma hepatocelular variedad fibrolamelar tratados con 5-FU e interferon alfa-2b. Cinco de 8 enfermos evaluados después del tratamiento presentaron una respuesta parcial o total.

Podemos concluir de esta revisión que la variedad fibrolamelar del carcinoma hepatocelular representa una situación clínica infrecuente que afecta a adolescentes y adultos jóvenes, es frecuentemente metastásica al momento del diagnóstico y su tratamiento es quirúrgico. El PET demostró ser un buen método de imágenes para realizar la etapificación inicial y para detectar en forma precoz focos de recidiva en el seguimiento. La sobrevida a largo plazo dependerá de la calidad de la resección, del estadio tumoral y de la presencia de compromiso linfonodal. Más de la mitad de los pacientes con sobrevidas alejadas recurrirán y requerirán de uno o más procedimientos quirúrgicos resectivos adicionales.

\section{REFERENCIAS}

1. Moreno-Luna L, Arrieta O, García-Leiva J, Martínez B, Torre A, Uribe $M$ ET AL. Clinical and pathologic factors associated with survival in young adult patients with fibrolamellar hepatocarcinoma. BMC Cancer 2005; 5; 142.

2 Berman $\mathrm{H}$, Burnham J, Sheahan D. Fibrolamellar carcinoma of the liver: an immunohistochemical study of nineteen cases and a review of the literature. Hum Pathol 1988; 19: 784-93.

3. Stipa F, Yoon S, Liau K, Fong Y, Jarnagin W, D'Angelica $M$ ET AL. Outcome of patients with fibrolamellar hepatocellular carcinoma. Cáncer 2006; 106: 1331-8.

4. Silva H, León G, NÁquira N. Carcinoma hepatocelular fibrolamelar. Presentación de 4 casos. Rev Méd Chile 1988; 116: 153-6.

5. Craig J, Peters R, Edmondson H, Omata M. Fibrolamellar carcinoma of the liver: a tumor of adolescents and young adults with distinctive clinic-pathologic features. Cáncer 1980; 46: 372-9.

6. Hemming A, Langer B, Sheiner P, Greig P, Tayor B. Aggressive surgical management of fibrolamellar hepatocellular carcinoma. J Gastrointest Surg 1997; 1: 342-6.

7. El-Serag H, Dávila J. Is fibrolamellar carcinoma different from hepatocellular carcinoma? A US population-based study. Hepatology 2004; 39: 798-803.

8. Katzenstein H, Krailo M, Malogolowkin M, Ortega J, Qu W, Douglass E ET aL. Fibrolamellar hepatocellular carcinoma in children and adolescents. Cáncer 2003; 97: 2006-12.

9. Ichikawa T, Federle M, Grazioli L, Madariaga J, NALESKIK M, MARSH W. Fibrolamellar hepatocellular carcinoma: imaging and pathologic findings in 31 recent cases. Radiology 1999; 213: 352-61.

10. Blachar A, Federle M, Ferris J, Lacomis J, Waltz J, ARmfield D ET AL. Radiologists' performance in the diagnosis of liver tumors with central scars by using specific CT criteria. Radiology 2002; 223: 532-9.

11. Massardo T, Jofré M, Sierralta P, Canessa J, González P, Humeres P et al. Análisis de 1.000 casos de estudios metabólicos en Chile con PET ${ }^{18}$ Flúor-deoxiglucosa (FDG). Rev Méd Chile 2007; 135: 375-83.

12. Soreide O, Czerniak A, Bradpiece $H$, Bloom $S$, Blumgart L. Characteristics of fibrolamellar hepatocellular carcinoma. A study of nine cases and a review of the literature. Am J Surg 1986; 151: 518-23.

13. Hirohashi S, Ishak K, Kojiro M, Wanless I, Theise N, Tsukuma H ET aL. Hepatocellular carcinoma. In World Health Organization Classification of Tumours. Pathology and Genetics of Tumours of the Digestive System. IARC Press; Lyon, 2000; 159-72.

14. Kakar S, Burgart L, Batts K, Garcia J, Jain D, Ferrell L. Clinicopathologic features and survival in fibrolamellar carcinoma: comparison with conventional hepatocellular carcinoma with and without cirrhosis. Mod Pathol 2005; 18: 1417-23.

15. Patt Y, Hassan M, Lozano R, Brown T, Vauthey J, CURLEy S ET AL. Phase II trial of systemic continuous fluorouracil and subcutaneous recombinant interferon Alfa-2b for treatment of hepatocellular carcinoma. J Clin Oncol 2003; 21: 421-7. 